\title{
Hospital Volume and Outcome after Bilateral Internal Mammary Artery Grafting
}

\author{
Andrea Perrotti, ${ }^{1}$ Daniel Reichart, ${ }^{2}$ Giuseppe Gatti, ${ }^{3}$ Giuseppe Faggian, ${ }^{4}$ Francesco Onorati, ${ }^{4}$ Marisa De Feo, \\ Sidney Chocron, MD,${ }^{1}$ Magnus Dalén, ${ }^{6}$ Giuseppe Santarpino, ${ }^{7}$ Antonino S. Rubino, ${ }^{8}$ Daniele Maselli, ${ }^{9}$ \\ Riccardo Gherli, ${ }^{10}$ Antonio Salsano, ${ }^{11}$ Francesco Nicolini, ${ }^{12}$ Marco Zanobini, ${ }^{13}$ Karl Bounader, ${ }^{14}$ Stefano Rosato, ${ }^{15}$ \\ Tuomas Tauriainen, ${ }^{16}$ Tatu Juvonen, ${ }^{17}$ Giovanni Mariscalco, ${ }^{18}$ Vito G. Ruggieri, ${ }^{19}$ Fausto Biancari ${ }^{16,20}$ \\ ${ }^{1}$ Department of Thoracic and Cardio-Vascular Surgery, University Hospital Jean Minjoz, Besançon, France; ${ }^{2}$ Hamburg University \\ Heart Center, Hamburg, Germany; ${ }^{3}$ Division of Cardiac Surgery, Ospedali Riuniti, Trieste, Italy; ${ }^{4}$ Division of Cardiovascular Surgery, \\ Verona University Hospital, Verona, Italy; ${ }^{5}$ Division of Cardiac Surgery, Universita degli Studi della Campania Luigi Vanvitelli, \\ Caserta, Italy; ${ }^{6}$ Department of Molecular Medicine and Surgery, Department of Cardiothoracic Surgery and Anesthesiology, \\ Karolinska Institutet, Karolinska University Hospital, Stockholm, Sweden; ${ }^{7}$ Cardiovascular Center, Paracelsus Medical University, \\ Nuremberg, Germany and Città di Lecce Hospital GVM Care\&Research, Lecce, Italy; ${ }^{8}$ Centro Clinico-Diagnostico "G.B. \\ Morgagni”, Centro Cuore, Pedara, Italy; ${ }^{9}$ Department of Cardiac Surgery, St. Anna Hospital, Catanzaro, Italy; ${ }^{10}$ Department of \\ Cardiovascular Sciences, Cardiac Surgery Unit, S. Camillo-Forlanini Hospital, Rome, Italy; ${ }^{11}$ Division of Cardiac Surgery, University \\ of Genoa, Genoa, Italy; ${ }^{12}$ Division of Cardiac Surgery, University of Parma, Parma, Italy; ${ }^{13}$ Department of Cardiac Surgery, Centro \\ Cardiologico - Fondazione Monzino IRCCS, University of Milan, Milan, Italy; ${ }^{14}$ Division of Cardiothoracic and Vascular Surgery, \\ Pontchaillou University Hospital, Rennes, France; ${ }^{15}$ National Center of Global Health, Istituto Superiore di Sanità, Rome, Italy; \\ ${ }^{16}$ Department of Surgery, Oulu University Hospital and University of Oulu, Oulu, Finland; ${ }^{17}$ Heart Center, Helsinki University \\ Hospital, Finland; ${ }^{18}$ Department of Cardiovascular Sciences, Clinical Sciences Wing, University of Leicester, Glenfield Hospital, \\ Leicester, UK; ${ }^{19}$ Division of Cardiac Surgery, Reims University Hospital, Reims, France; ${ }^{20}$ Heart Center, Turku University Hospital, \\ and Department of Surgery, University of Turku, Turku, Finland
}

\section{ABSTRACT}

Background: Bilateral internal mammary artery (BIMA) grafting largely is underutilized in patients undergoing coronary artery bypass grafting (CABG), partly because of the perceived increased complexity of the procedure.

Aims: In this study, we evaluated whether BIMA grafting can safely be performed also in centers, where this revascularization strategy infrequently is adopted.

Methods: Out of 6,783 patients from the prospective multicenter E-CABG study, who underwent isolated nonemergent CABG from January 2015 to December 2016, 2,457 underwent BIMA grafting and their outcome was evaluated in this analysis.

Results: The mean number of BIMA grafting per center was 82 cases/year and hospitals were defined as high or low volume, according to this cutoff value. Six hospitals were considered as centers with a high volume of BIMA grafting (no. of procedures ranging from 120 to 267/year; overall: 2,156; prevalence: $62.2 \%$ ) and nine hospitals as centers with a low volume of BIMA grafting (no. of procedures ranging from 2 to 39/year; overall: 301; prevalence: $9.1 \%$ ). Multilevel mixed-effects regression analysis

Received September 24, 2019; accepted April 10, 2020.

Correspondence: Dr: Andrea Perrotti, Department of Thoracic and CardioVascular Surgery, University Hospital Jean Minjoz, Besançon, France (e-mail: a.perrotti@hotmail.it). showed that the low- and high-volume cohorts had similar outcomes. Propensity score one-to-one matching analysis of 292 pairs showed that the low-volume cohort had a significantly shorter intensive care unit stay $(2.2 \pm 2.3$ versus $2.9 \pm 4.8$ days, $P=.020)$. The rates of in-hospital death $(1.0 \%$ versus $0.3 \%, P=.625)$, deep sternal wound infection/mediastinitis $(3.8 \%$ versus $3.1 \%, P=.824)$, and 1-year survival $(98.1 \%$ versus $99.7 \%, P=.180)$ as well as other outcomes were similar between the high- and low-volume cohorts.

Conclusions: BIMA grafting can be safely performed also in centers in which this revascularization strategy is infrequently performed.

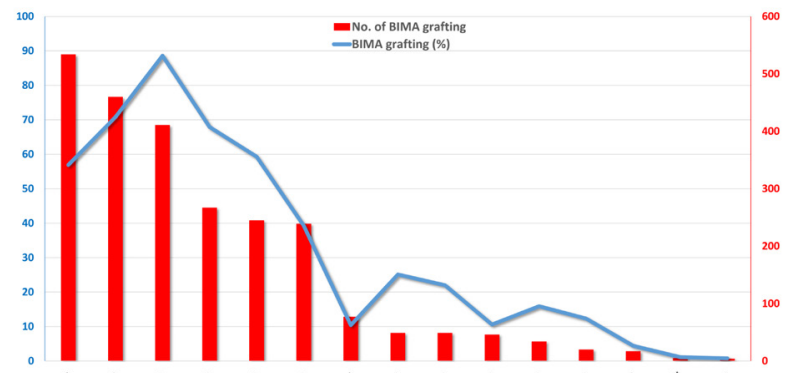

Prevalence of bilateral internal mammary artery grafting in different European cardiac centers. 
Table 1. Baseline characteristics

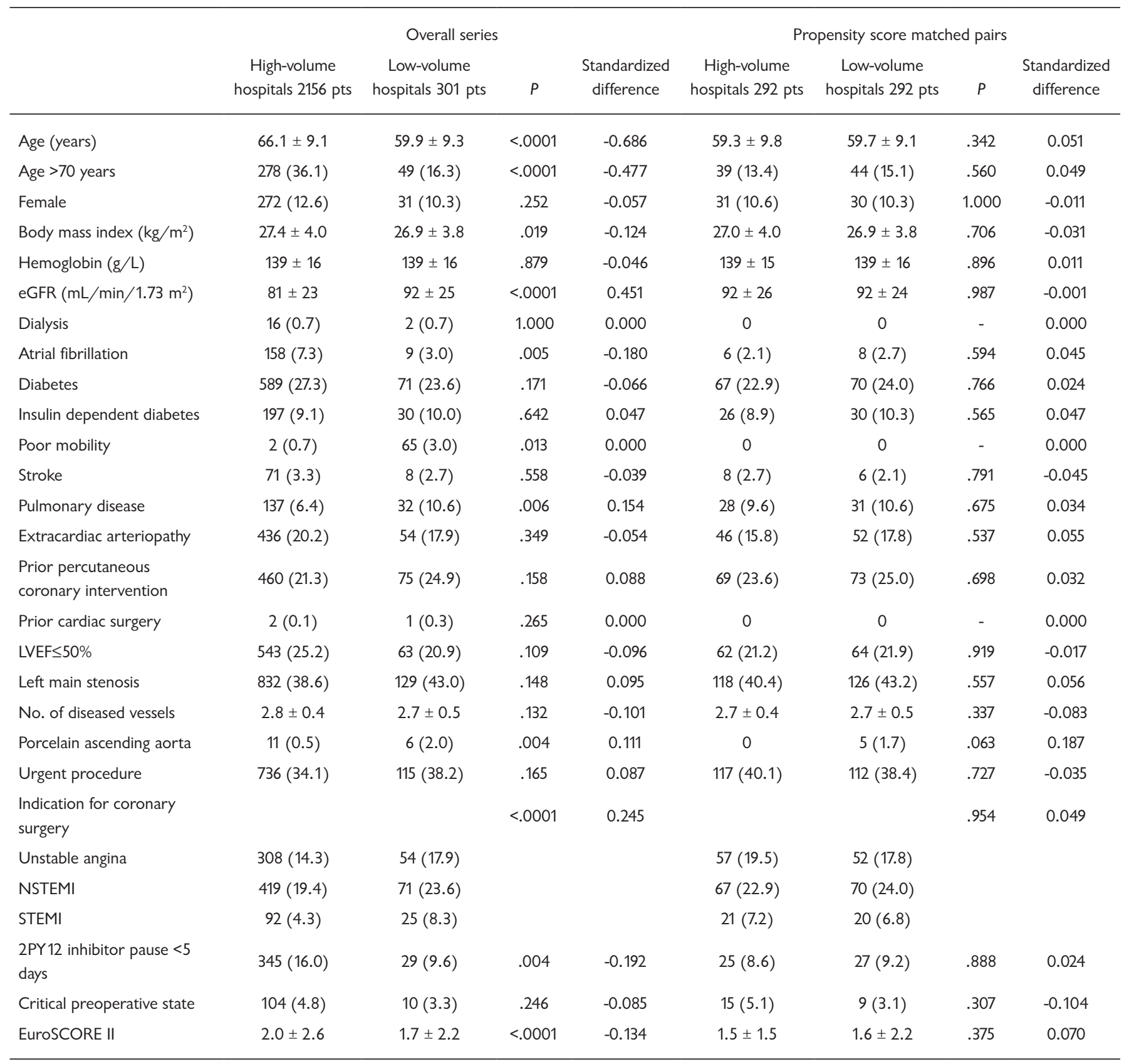

Continuous variables are reported as means and standard deviation and nominal variables as counts and percentages. Clinical variables are according to the EuroSCORE II definition criteria. eGFR: glomerular filtration estimated according to the MDRD equation; LVEF: left ventricular ejection fraction; NSTEMI: non ST-elevation myocardial infarction; STEMI: ST-elevation myocardial infarction; IABP: intra-aortic balloon pump.

\section{INTRODUCTION}

Several studies showed improved long-term outcomes regarding survival, graft patency, and adverse cardiac events after isolated coronary artery bypass grafting (CABG) using bilateral internal mammary artery (BIMA) grafts compared with single internal mammary artery graft [Dorman 2012; Weiss
2013; Iribarne 2017]. The use of BIMA grafting varies considerably between centers, and this bypass grafting strategy likely is underutilized in many centers owing to the perceived complexity of BIMA grafting compared with single internal mammary artery grafting [LaPar 2015; Iribarne 2017]. It is unknown if a low center volume of BIMA grafting carries a risk for worse outcomes after this procedure. In this prospective multicenter 


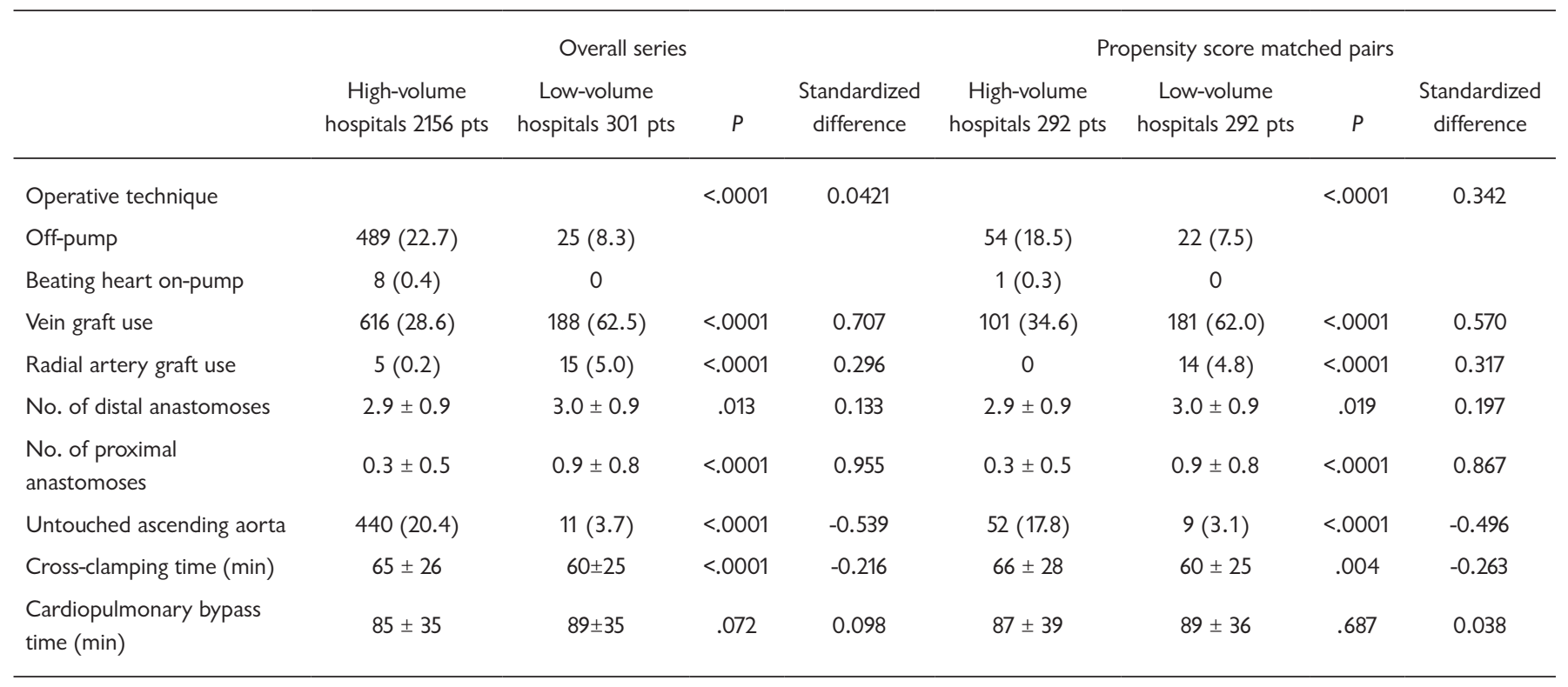

Continuous variables are reported as mean and standard deviation. Categorical variables are reported as absolute number and percentages (in parentheses).

registry, we evaluated whether this revascularization can be safely performed in centers with a low volume of BIMA grafting.

\section{METHODS}

Patient population and data collection: The European Coronary Artery Bypass Grafting (E-CABG) registry is a prospective, multicenter study enrolling patients undergoing isolated CABG at 16 European centers of cardiac surgery (Besançon, France; Catanzaro, Italy; Genoa, Italy; Hamburg, Germany; Leicester, UK; Milan, Italy; Nuremberg, Germany; Naples, Italy; Oulu, Finland; Parma, Italy; Pedara, Italy; Rennes, France; Rome, Italy; Stockholm, Sweden; Trieste, Italy; Verona, Italy). This study is registered in Clinicaltrials.gov (Identifier: NCT02319083). The study protocol and definition criteria of baseline, operative, and postoperative variables are described elsewhere [Biancari 2015]. The Institutional Review Board or Ethical Committee of the participating centers approved this study.

Out of 7,352 consecutive patients operated on from January 2015 to May 2017 and enrolled in the E-CABG registry, 53 patients were excluded because their data were retrieved from one participating center only during the last year of recruitment. Further, 181 patients were excluded because they were operated on at two participating centers after the end of the official period of recruitment. Threehundred-thirty-five patients underwent emergency surgery and were excluded from this analysis.

Overall, 6,783 patients underwent isolated elective or urgent CABG. Among these patients, 2,457 patients $(36.2 \%)$ underwent BIMA grafting and were the subjects of this comparative analysis. The outcome of patients operated in centers with high-volume BIMA grafting was compared with that of patients operated in centers with a low volume of BIMA grafting. Centers were classified as high or low BIMA grafting volume centers, depending on the total number of BIMA procedures performed per year. Centers performing more than the mean yearly number of BIMA grafting procedures in the overall series were classified as high-volume centers, and centers performing less than this mean number were defined as low-volume centers.

Outcome end-points: In-hospital death and sternal wound infection (SWI) were the primary end-points of this study. Secondary end-points were the need of prolonged inotropic support, postoperative use of intra-aortic balloon pump (IABP) or extracorporeal membrane oxygenation (ECMO), stroke, acute kidney injury, the number of transfused red blood cell units, reoperation for bleeding as well as length of stay in the intensive care unit and in the hospital.

The definition criteria of these end-points are reported in detail elsewhere [Biancari 2015]. The diagnosis and severity of SWI was defined and graded, according to the Centers for Disease Control and Prevention classification of the surgical site infections [Mangram 1999]. The severity of postoperative acute kidney injury was classified according to the Kidney Disease: Improving Global Outcomes (KDIGO) criteria [Work Group - KDIGO 2013].

Statistical analysis: Statistical analyses were performed using the SPSS v. 24.0 (IBM Corporation, New York, NY), SAS v. 9.4 (SAS Institute Inc., Cary, NC) and Stata v. 14.2 (StataCorp LLC, College Station, Texas) statistical software. Mann-Whitney U-test, Fisher's exact test, Chi-square test, and Kruskal-Wallis tests were used for univariate analysis in the unmatched population. Multilevel mixed-effects linear and logistic regression methods were used for institutional-volume 
Table 3. Outcomes

\begin{tabular}{|c|c|c|c|c|c|c|c|}
\hline & \multicolumn{4}{|c|}{ Overall series } & \multicolumn{3}{|c|}{ Propensity score matched pairs } \\
\hline Prolonged inotropic support & $519(24.1)$ & $83(27.6)$ & $<.0001$ & $1.39(0.19-10.28)$ & $71(24.3)$ & $81(27.7)$ & .391 \\
\hline Intra-aortic balloon pump & $55(2.6)$ & $9(3.0)$ & .654 & $0.58(0.16-2.05)$ & $8(2.7)$ & $9(3.1)$ & 1.000 \\
\hline Stroke & $17(0.7)$ & $2(0.7)$ & 1.000 & $1.20(0.26-5.63)$ & $2(0.7)$ & $2(0.7)$ & 1.000 \\
\hline $\begin{array}{l}\text { Sternal wound infection - } \\
\text { overall }\end{array}$ & $140(6.5)$ & $28(9.3)$ & .071 & $0.49(0.22-1.10)$ & $18(6.2)$ & $23(7.9)$ & .522 \\
\hline E-CABG bleeding grade 2-3 & $102(4.7)$ & $10(3.3)$ & .272 & $1.24(0.56-2.75)$ & $12(4.1)$ & $10(3.4)$ & .832 \\
\hline No. of transfused RBC units & $0.9 \pm 1.7$ & $0.8 \pm 1.6$ & .296 & $-0.84(-0.41-0.24)$ & $0.8 \pm 1.6$ & $0.7 \pm 1.4$ & .418 \\
\hline Resternotomy for bleeding & $62(2.9)$ & $6(2.0)$ & .382 & $1.48(0.61-3.58)$ & $8(2.7)$ & $6(2.1)$ & .791 \\
\hline Intensive care unit stay (days) & $3.1 \pm 3.7$ & $2.2 \pm 2.3$ & $<.0001$ & $0.52(1.026-1.31)$ & $2.9 \pm 4.8$ & $2.2 \pm 2.3$ & .020 \\
\hline In-hospital stay (days) & $10.2 \pm 7.0$ & $9.6 \pm 5.7$ & .005 & $0.07(-2.21-2.34)$ & $10.6 \pm 11.1$ & $9.6 \pm 5.7$ & .169 \\
\hline In-hospital death & $33(1.5)$ & $1(0.3)$ & .114 & $3.80(0.44-32.72)$ & $3(1.0)$ & $1(0.3)$ & .625 \\
\hline 1-year survival (\%) & 97.5 & 99.7 & .023 & $5.99(0.79-43.71)$ & 98.1 & 99.7 & .180 \\
\hline
\end{tabular}

Continuous variables are reported as the mean and standard deviation. Categorical variables are reported as counts and percentages (in parentheses). ECMO: extracorporeal membrane oxygenation; Acute kidney injury: estimated according to the KDIGO criteria; E-CABG bleeding grade 2-3: resternotomy for bleeding or RBC transfused $>4$ units; RBC: red blood cell; *: estimates are odds ratio or coefficients with $95 \%$ confidence interval.

adjusted analysis of the impact of high- compared with lowvolume BIMA grafting on the outcomes as adjusted by age, gender, body mass index, estimated glomerular filtration rate, pulmonary disease, stroke, poor mobility, extracardiac arteriopathy, diabetes, left ventricular ejection fraction, previous percutaneous coronary intervention, indication for revascularization, urgent operation, critical preoperative status, left main coronary artery stenosis, number of diseased vessels, and revascularization technique.

Propensity score one-to-one matching analysis was performed to adjust for covariates difference between patients operated on at centers with high- and low-volume BIMA grafting. A propensity score was estimated, using the abovementioned covariates in logistic regression with the high- and low-volume BIMA grafting centers as a dependent variable. Propensity score matching was performed using the nearest neighbour method and a caliper of 0.2 of the standard deviation of the logit of the propensity score. The t-test for paired samples for continuous variables, the McNemar test for dichotomous variables, and the analysis of the standardized differences after matching were used to evaluate the balance between the matched groups. A standardized difference lower than 0.10 was considered as an acceptable imbalance between the study groups. The t-test for paired samples and $\mathrm{McNemar}$ test were employed to evaluate any difference in the early outcomes of propensity score matched pairs. Survival analyses were performed using the Kaplan-Meier method and log-rank test. The Cox frailty survival model with gamma distribution was employed for multilevel adjusted survival analysis. The Kaplan-Meier method with the Klein-Moeschberger stratified log-rank test was used to evaluate the differences in survival of propensity score-matched pairs. All tests were two-sided and $P<.05$ was set for statistical significance.

\section{RESULTS}

Outcomes in the overall series: The prevalence of BIMA grafting at the participating centers varied from $0.8 \%$ to $88.6 \%$. The number and proportion of BIMA grafting in each participating center are summarized in Figure 1. The mean number of BIMA grafting per center was 82 patients/year (median: 24.5 patients/ year), and the participating hospitals were defined as high- or low-volume BIMA grafting centers, according to this cutoff value. 
Accordingly, six hospitals were considered as centers with a high volume of BIMA grafting (overall: 2,156 patients; no. of patients/center ranging from 119.5 to 267.0 per year; mean, $179.5 \pm 63.0$ cases/year; prevalence ranging from $39.3 \%$ to $88.6 \%$; overall prevalence: $62.2 \%$ ) and nine hospitals as centers with a low volume of BIMA grafting (overall: 301 patients; no. of patients/center ranging from 2.0 to 38.5 per year; mean, $16.7 \pm 12.1$ cases/year; prevalence ranging from $0.8 \%$ to $25.1 \%$; overall prevalence: $9.1 \%$ ).

The baseline characteristics and operative variables of the study cohorts are summarized in Tables 1 and 2. Patients in the high-volume cohort had a significantly higher operative risk (mean EuroSCORE II, $2.0 \pm 2.6$ versus $1.7 \pm 2.2 \%$, median, 1.3 versus $1.1 \%, P<.0001$ ), mainly driven by the significantly higher age of patients operated on at these centers $(66.1 \pm 9.1$ versus $59.9 \pm 9.3$ years, $P<.0001)$. In fact, the prevalence of patients aged $>70$ years in the high-volume cohort was $36.1 \%$ compared with $16.3 \%$ in the low-volume $\operatorname{cohort}(P<.0001)$. On the other hand, the low-volume cohort had a higher prevalence of acute coronary syndrome, pulmonary disease, and porcelain aorta. Vein grafts $(62.5 \%$ versus $28.6 \%, P<.0001)$ and radial artery graft $(5.0 \%$ versus $0.2 \%$, $P<.0001)$ were used more frequently in the low-volume cohort. Furthermore, the ascending aorta was left untouched more frequently in the high-volume cohort $(20.4 \%$ versus $3.7 \%, P<.0001)$ compared with the low-volume cohort.

Multilevel mixed-effects regression analyses showed the low-volume cohort had similar hospital outcomes and 1-year survival compared with the high-volume cohort. The rates of in-hospital death $(1.5 \%$ versus $0.3 \%$, adjusted OR 3.80 , 95\% CI 0.44-32.72) and deep sternal wound infection/mediastinitis (3.1\% versus $4.3 \%$, adjusted OR 0.62 , 95\% CI 0.19 2.03) were similar in the study cohorts (Table 3).

Propensity score matched analysis: One-to-one propensity matching provided 292 pairs with similar baseline characteristics as confirmed by standardized differences being lower than 0.10 for all variables but the prevalence of porcelain, which tended to be higher in the low-volume cohort (Table 1). The use of vein and radial artery grafting was higher in the low-volume BIMA cohort. The number of distal anastomoses were higher in the low-volume cohort compared with the high-volume BIMA cohort (Table 2).

Paired tests showed that the low-volume cohort had a significantly shorter intensive care unit stay $(2.2 \pm 2.3$ versus $2.9 \pm$ 4.8 days, $P=.020)$. Otherwise, the propensity score-matched cohorts had similar early and 1-year outcomes (Table 3). As in the overall cohort, rates of in-hospital death $(1.0 \%$ versus $0.3 \%, P=.625)$, deep sternal wound infection/mediastinitis (3.8\% versus $3.1 \%, P=.824)$, and 1 -year survival $(98.1 \%$ versus $99.7 \%, P=.180$ ) were similar between the high- and low-volume cohorts (Table 3 ).

\section{DISCUSSION}

In this multicenter study, we observed that: 1) a low volume of BIMA grafting was not associated with a higher mortality or morbidity; 2) BIMA grafting was underutilized in about two-thirds of the centers participating in this registry; 3) patients undergoing BIMA grafting at low-volume centers were younger and with lower operative risk than those operated at high-volume centers; 4) low-volume centers utilized vein grafts and radial artery grafts more frequently than high-volume centers.

This analysis did not take into account the individual surgeon's impact on the outcomes, but the rather low proportion of BIMA grafting in low-volume hospitals suggests also a low volume of BIMA grafting for individual surgeons at these institutions.

These results are of clinical importance in view of the increased risk of early adverse events and the uncertainty over the real long-term benefits of BIMA grafting. In fact, despite several large studies that demonstrated the benefits of this revascularization strategy [Dorman 2012; Weiss 2013; Iribarne 2017; Lytle 2004], a number of recent studies with very long follow-up data failed to demonstrate the benefit of the BIMA grafting strategy [Dalén 2014; Garatti 2014; Pevni 2017] compared with single internal mammary artery grafting associated with vein grafts. In particular, a study from the Swedish Web System for Enhancement and Development of Evidence-Based Care in Heart Disease Evaluated According to Recommended Therapies (SWEDEHEART] with solid late survival data posed doubts about the long-term benefits of BIMA grafting [Dalén 2014]. Still, this study was not randomized, and revascularization with BIMA grafts was performed only in $1.1 \%$ of patients. The higher risk of severe sternal wound infection as well as the increased operative time and costs associated with BIMA grafting have led to a hesitation to implement this grafting strategy [Gray 2017; Taggart 2019]. Only one randomized trial has been performed, showing an increased risk for sternal wound infection with BIMA grafting, but similar 10-year outcome [Taggart 2019].

BIMA grafting technically is considered more demanding than single internal mammary artery grafting using vein or radial arterial grafts as secondary conduits. Indeed, coronary artery anatomy and severity of coronary artery disease call for a detailed planning of the BIMA grafting strategy to achieve complete myocardial revascularization. Harvesting of both internal mammary arteries and configuration of arterial grafts, according to the coronary artery anatomy, make the operation more complex and thereby require longer operative times and may expose patients to increased intraoperative blood loss compared with single internal mammary artery grafting. Therefore, hospital volume and individual surgeon experience may affect the early and late outcome after BIMA grafting. A recent study showed that BIMA grafting can safely be performed by residents with early and 1-year outcomes similar to that of more experienced surgeons [Pasrija 2018]. However, the small size of this study and lack of adjusted analyses prevented conclusive results on the safety of BIMA grafting, when performed by less experienced surgeons.

Since experience of the surgeon and hospital volume seem to significantly affect the postoperative outcome after more complex CABG procedures, such as off-pump coronary surgery [Benedetto 2018], an inter-institutional analysis of the outcome after BIMA grafting is expected to provide important insights on the potential risks of BIMA grafting utilization in 
low-volume BIMA centers. However, the present analysis did not show higher risk of mortality and morbidity in centers with limited experience with this technique compared with centers with large experience.

These results should be viewed in light of an evident disparity in the adoption of BIMA grafting between the centers participating in this prospective European registry. Indeed, the prevalence of BIMA grafting was only $9 \%$ in low-volume centers, whilst it was $62 \%$ in high-volume centers. Lowvolume centers most likely have been using BIMA grafting as a bail-out strategy as indicated by the higher prevalence of porcelain aorta in this cohort. The different use of vein graft and radial artery grafts between low- and high-volume BIMA centers suggest that low-volume centers were more prone to adopt grafts other than the internal mammary artery to accomplish a complete revascularization. Despite these differences, the early and 1-year postoperative outcomes were similar after propensity score matching. These findings suggest that, despite its technically more demanding nature compared with single internal mammary artery, BIMA grafting can safely be performed also in hospitals with limited experience with this revascularization method.

Surgeons resort to the use of BIMA grafting either by choice or necessity. In the presence of a diffusely atherosclerotic ascending aorta that precludes cannulation, clamping, or both, BIMA use, with both conduits in situ or as composite conduit, yields potentialities of off-pump (and on-pump beating heart) technique for myocardial revascularization; besides, the proximal stem of the right (or left) BIMA also might be a useful inflow for a saphenous vein or radial artery coronary graft [Benussi 2019]. The lack of other suitable coronary grafts, such as the case of a prior bilateral total saphenectomy or a Doppler Allen's test positive for ischemia of the dominant arm, may be more examples of when BIMA use is needed, regardless of surgical priority and the patient's age and preoperative conditions. On the other hand, surgeons confident in the long-term benefits of BIMA grafting may choose to adopt it for patients aged 70-75 or younger, regardless of the expected risk of sternal wound infections, as well as for older subjects with low risk of sternal complications. Obviously, the choice becomes more challenging in the presence of one or more out of the following recognized predictors of sternal wound infections after CABG: female sex, morbid obesity, diabetes mellitus (especially when insulin-dependent and/or with poor glycemic control), chronic obstructive pulmonary disease, extracardiac arteriopathy, chronic dialysis, congestive heart failure, and urgent surgical priority [Gatti 2016].

\section{LIMITATIONS}

The E-CABG registry is a prospective multicenter registry, with clear definition criteria, which guarantee the uniformity of collected data. Still, this study has a few limitations. First, this is not a randomized trial, and data might possibly be biased by inter-institutional differences. Although we adjusted for such potential differences at patient and hospital level using multilevel mixed-effects regression methods, the study design does not guarantee uniformity in patient selection, operative experience, and perioperative care. Second, this study lacks details on the harvesting technique and graft configuration, which may have a potential impact on the outcomes. Third, the short follow up of this series prevents any conclusive result on a possible impact of the hospital volume on the late outcome after BIMA grafting. Fourth, despite the rather large size of this series, we were able to match only 292 pairs of patients, due to the limited number of patients operated on at low-volume centers. Finally, individual surgeon data was not collected in the E-CABG registry, and therefore we are not able to perform a surgeon-volume analysis. However, the latter analysis might be prevented by the rather limited number of patients undergoing BIMA grafting in low-volume hospitals, which also suggests a low volume of BIMA grafting for individual surgeons.

\section{CONCLUSIONS}

The present results suggest that increasing the experience with BIMA grafting does not translate into an improvement of early outcomes of patients undergoing non-emergent CABG. These findings indicate that when more conclusive results on its long-term benefits are gathered, BIMA grafting strategy could safely be implemented in hospitals with limited experience with this revascularization technique.

\section{REFERENCES}

Benedetto U, Lau C, Caputo M, Kim L, Feldman DN, Ohmes LB, et al. 2018. Comparison of outcomes for off-pump versus on-pump coronary artery bypass grafting in low-volume and high-volume centers and by low-volume and high-volume surgeons. Am J Cardiol 121:552-557.

Benussi B, Gatti G, Gripshi F, Biondi F, Porcari A, Ruggiero D, et al. 2019. Clinical Validation of a Coronary Surgery Technique That Minimizes Aortic Manipulation. Ann Thorac Surg 107:1166-1173.

Biancari F, Ruggieri VG, Perrotti A, Svenarud P, Dalén M, Onorati F, et al. 2015. European multicenter study on coronary artery bypass grafting (E-CABG registry): study protocol for a prospective clinical registry and proposal of classification of postoperative complications. J Cardiothorac Surg 10:90.

Dalén M, Ivert T, Holzmann MJ, Sartipy U. 2014. Bilateral versus single internal mammary coronary artery bypass grafting in Sweden from 19972008. PLoS One 9:e86929.

Dorman MJ, Kurlansky PA, Traad EA, Galbut DL, Zucker M, Ebra G. 2012. Bilateral internal mammary artery grafting enhances survival in diabetic patients. A 30-year follow-up propensity-score matched cohorts. Circulation 126:2935-2942.

Garatti A, Castelvecchio S, Canziani A, Corain L, Generali T, Mossuto E, et al. 2014. Long-term results of sequential vein coronary artery bypass grafting compared with totally arterial myocardial revascularization: a propensity score-matched follow-up study. Eur J Cardiothorac Surg 46:1006-13.

Gatti G, Dell'Angela L, Barbati G, Benussi B, Forti G, Gabrielli M, et al. 2016. A predictive scoring system for deep sternal wound infection after bilateral internal thoracic artery grafting. Eur J Cardiothorac Surg 49:910-917. 
Gray AM, Murphy J, Altman DG, Benedetto U, Campbell H, Flather M, et al. 2017. One-year costs of bilateral or single internal mammary grafts in the Arterial Revascularization Trial. Heart. 103:1719-1726.

Iribarne A, Goodney PP, Flores AM, DeSimone J, DiScipio AW, et al. 2017. National trends and geographic variation in bilateral internal mammary artery use in the United States. Ann Thorac Surg 104:1902-1907.

Iribarne A, Schmoker JD, Malenka DJ, Leavitt BJ, McCullough JN, Weldner PW, et al. 2017. Does use of bilateral internal mammary artery grafting reduce long-term risk of repeat coronary revascularization? A multicenter analysis. Circulation 136:1676-1685.

Kidney Disease: Improving Global Outcomes (KDIGO) CKD Work Group. 2013. KDIGO 2012 Clinical practice guideline for the evaluation and management of chronic kidney disease. Kidney Inter Suppl 3:1-150.

LaPar DJ, Crosby IK, Rich JB, Quader MA, Speir AM, Kern JA, et al. 2015. Bilateral internal mammary artery use for coronary artery bypass grafting remains underutilized: a propensity-matched multi-institution analysis. Ann Thorac Surg 100:8-14.

Lytle BW, Blackstone EH, Sabik JF, Houghtaling P, Loop FD, Cosgrove
DM. 2004. The effect of bilateral internal thoracic artery grafting on survival during 20 postoperative years. Ann Thorac Surg 78:2005-12.

Mangram AJ, Horan TC, Pearson ML, Silver LC, Jarvis WR. 1999. Guideline for prevention of surgical site infection. Hospital Infection Control Practices Advisory Committee. Infect Control Hosp Epidemiol 20:250-278.

Pasrija C, Ghoreishi M, Shah A, Rouse M, Gammie JS, Kon ZN, Taylor BS. 2018. Bilateral internal mammary artery use can be safely taught without increasing morbidity or mortality. Ann Thorac Surg 105:76-82.

Pevni D, Ben-Gal Y, Mohr R, Ganiel A, Paz Y, Kramer A, et al. 2017. One or two internal thoracic grafts? Long-term follow-up of 957 offpump coronary bypass surgeries. Ann Thorac Surg 104:70-77.

Taggart DP, Benedetto U, Gerry S, Altman DG, Gray AM, Lees B, et al. 2019. Bilateral versus single internal-thoracic-artery grafts at 10 years. N Engl J Med 380:437-446.

Weiss AJ, Zhao S, Tian DH, Taggart DP, Yan TD. 2013. A meta-analysis comparing bilateral internal mammary artery with left internal mammary artery for coronary artery bypass grafting. Ann Cardiothorac Surg 2:390-400 